\title{
A Sustainable Approach to Waste-Minimized Sonogashira Cross-Coupling Reaction Based on Recoverable/Reusable heterogeneous Catalytic/Base System and Acetonitrile Azeotrope
}

Vadym Kozell, ${ }^{[a]}$ Michael McLaughlin, ${ }^{[a]}$ Giacomo Strappaveccia, ${ }^{[a]}$ Stefano Santoro, ${ }^{[a]}$ Lucia Anna Bivona, ${ }^{[b],[c]}$ Carmela Aprile, ${ }^{[c]}$ Michelangelo Gruttadauria, ${ }^{[b]}$ Luigi Vaccaro ${ }^{*[a]}$

[a] Vadym Kozel, Michael McLaughlin, Dr. Giacomo Strappaveccia, Prof. Dr. L. Vaccaro CEMIN - Dipartimento di Chimica, Biologia e Biotecnologie, Università di Perugia Via Elce di Sotto, 8; Perugia, Italia. E-mail: luigi.vaccaro@unipg.it

[b] Dr. Lucia Bivona, Prof. Dr. Michelangelo Gruttadauria Dipartimento di Scienze e Tecnologie Biologiche, Chimiche e Farmaceutiche (STEBICEF)

Sez. di Chimica, Università di Palermo, Viale delle Scienze s/n, Ed. 17, 90128 Palermo, Italy.

[c] Dr. Lucia Bivona, Prof. Dr. Carmela Aprile Laboratory of Applied Material Chemistry (CMA), University of Namur, 61 rue de Bruxelles, 5000 Namur, Belgium.

Table of contents

General information

Typical procedures S2

E-factor calculations S4 - S5

Representative characterization data for catalyst $\mathbf{1}$ S6 - S7

Characterization data

$\mathrm{S} 8$ - S20 


\section{General information}

Unless otherwise stated, all solvents and reagents were used as obtained from Sigma-Aldrich Co. without further purification. GC-EIMS analyses were carried out by using a Hewlett-Packard HP 6890N Network GC system/5975 Mass Selective Detector equipped with an electron impact ionizer at $70 \mathrm{eV}$. NMR spectra were recorded on a Bruker DRX-ADVANCE $400 \mathrm{MHz}\left({ }^{1} \mathrm{H}\right.$ at $400 \mathrm{MHz},{ }^{13} \mathrm{C}$ at $100.6 \mathrm{MHz}$ and ${ }^{19} \mathrm{~F}$ at $376.4 \mathrm{MHz}$ ) in $\mathrm{CDCl}_{3}$ using TMS as the internal standard. Elemental Analyses were conducted on a Fisons EA1108CHN. Melting points are not corrected and they were measured on a Büchi 510. An Inductive Coupled Plasma-Optical Emission Spectrometer (ICP-OES 710 Agilent Technology) was used to determine the amount of leached palladium into the reaction products.

\section{Typical procedure for the Sonogashira reaction in $\mathrm{CH}_{3} \mathrm{CN} / \mathrm{H}_{2} \mathrm{O}$ azeotrope using homogeneous base ( $N$-methylpiperazine)}

1. The reaction was performed in a screw-capped vial with a magnetic stirrer. Catalyst (1) (10\% wt. of Pd) (4 mg, $0.4 \mathrm{mmol} \%), \mathrm{CH}_{3} \mathrm{CN} / \mathrm{H}_{2} \mathrm{O}$ azeotrope (0.94 mL, 84/16 wt\%), 1-methylpiperazine (2) (114 $\mathrm{mg}, 1.13 \mathrm{mmol}, 99 \%$ purity $)$, aryl iodide $(0.94 \mathrm{mmol})$, alkyne $(1.41 \mathrm{mmol})$ were consequently added and the resulting mixture was purged with $\mathrm{N}_{2}$ gas for 5 minutes under stirring. The resulting mixture was left under stirring at $90^{\circ} \mathrm{C}$.

2. After reaction completion, the reaction mixture was centrifuged for $10 \mathrm{~min}(5000 \mathrm{rpm})$ and the liquid was separated by decantation. $1 \mathrm{~mL}$ of acetonitrile/water azeotrope was added and the mixture was stirred for $5 \mathrm{~min}$, centrifuged for $10 \mathrm{~min}(5000 \mathrm{rpm})$ and the liquid was separated by decantation. Ethyl acetate $(5 \mathrm{~mL})$ was added and the organic layer was washed with water $(3 \times 1.5 \mathrm{~mL})$, dried over anhydrous $\mathrm{Na}_{2} \mathrm{SO}_{4}$ and the solvent was removed under vacuum to yield the product.

3. The catalyst was dried under nitrogen at $50^{\circ} \mathrm{C}$ and reused for the next catalytic cycle where reactants, reaction medium and 1-methylpiperazine were added.

\section{Typical procedure for the Sonogashira reaction in $\mathrm{CH}_{3} \mathrm{CN} / \mathrm{H}_{2} \mathrm{O}$ azeotrope using heterogeneous base (PS-piperazine, 8)}

1. The reaction was performed in a screw-capped vial with a magnetic stirrer. Catalyst (1) (10\% wt. of Pd) (4 mg, $0.4 \mathrm{mmol} \%), \mathrm{CH}_{3} \mathrm{CN} / \mathrm{H}_{2} \mathrm{O}$ azeotrope (0.94 mL, 84/16 wt\%), PS-piperazine (8) (335 mg, $1.13 \mathrm{mmol}, 3.37 \mathrm{mmol} / \mathrm{g})$, aryl iodide $(0.94 \mathrm{mmol})$, alkyne $(1.41 \mathrm{mmol}$,) were consequently added and the resulting mixture was purged with $\mathrm{N}_{2}$ gas for 5 minutes under stirring. The resulting mixture was left under stirring at $90^{\circ} \mathrm{C}$.

2. After reaction completion, $1 \mathrm{~mL}$ of acetonitrile/water azeotrope was added and the reaction mixture was stirred for $5 \mathrm{~min}$, centrifuged for $2 \mathrm{~min}(5000 \mathrm{rpm})$ and the liquid was separated by decantation. Than $1 \mathrm{~mL}$ more of acetonitrile water azeotrope was added to the catalyst-base mixture and stirred for $2 \mathrm{~min}$, centrifuged for $2 \mathrm{~min}(5000 \mathrm{rpm})$ and the liquid was separated by decantation. Resulting liquid phases were combined and the solvent was removed under vacuum to yield the product.

3. 1-Methylpiperazine (143 mg, $1.41 \mathrm{mmol}, 99 \%$ purity) in $1 \mathrm{~mL}$ of acetonitrile/water azeotrope was added to the catalyst-base mixture, stirred and after $1 \mathrm{~h}$ centrifuged for $2 \mathrm{~min}(5000 \mathrm{rpm})$ and the liquid phase was separated by decantation. Then $1 \mathrm{~mL}$ more of acetonitrile water azeotrope was added to the catalyst-base mixture was stirred for $2 \mathrm{~min}$, centrifuged for $2 \mathrm{~min}(5000 \mathrm{rpm})$ and the liquid was separated by decantation. The catalyst-base mixture was dried under vacuum at $50^{\circ} \mathrm{C}$ and reused for the next catalytic cycle where just reactants and reaction medium were added.

\section{Hg-Poisoning test}

The reaction was performed in a screw-capped vial with a magnetic stirrer. Catalyst (1) (10\% wt. of Pd) (4 mg, $0.4 \mathrm{mmol} \%$ ), $\mathrm{CH}_{3} \mathrm{CN} / \mathrm{H}_{2} \mathrm{O}$ azeotrope (0.94 mL, 84/16 wt\%), PS-piperazine (8) (335 mg, 1.13 
mmol, $3.37 \mathrm{mmol} / \mathrm{g}$ ), 4'-iodoacetophenone (231 mg, $0.94 \mathrm{mmol})$, phenylacetylene (144 mg, $1.41 \mathrm{mmol}$,) were consequently added and the resulting mixture was purged with $\mathrm{N}_{2}$ gas for 5 minutes under stirring. The resulting mixture was left under stirring at $90{ }^{\circ} \mathrm{C}$ until $60 \%$ conversion was reached. Then, 100 equivalents (relative to $\mathrm{Pd}$ ) of $\mathrm{Hg}(0)$ were added and the reaction was kept under stirring at $90{ }^{\circ} \mathrm{C}$ for additional 25 hours. After this time the reaction reached $62 \%$ conversion.

\section{Hot-filtration test}

The reaction was performed in a screw-capped vial with a magnetic stirrer. Catalyst (1) (10\% wt. of Pd) (4 mg, $0.4 \mathrm{mmol} \%$ ), $\mathrm{CH}_{3} \mathrm{CN} / \mathrm{H}_{2} \mathrm{O}$ azeotrope (0.94 mL, 84/16 wt\%), PS-piperazine (8) (335 mg, 1.13 mmol, $3.37 \mathrm{mmol} / \mathrm{g}$ ), 4'-iodoacetophenone (231 mg, $0.94 \mathrm{mmol}$ ), phenylacetylene (144 mg, $1.41 \mathrm{mmol}$,) were consequently added and the resulting mixture was purged with $\mathrm{N}_{2}$ gas for 5 minutes under stirring. The resulting mixture was left under stirring at $90^{\circ} \mathrm{C}$ until $60 \%$ conversion was reached. Then, the reaction mixture was filtered through a pre-heated sintered glass filter with the aid of Celite. Fresh PSpiperazine (8) $\left(335 \mathrm{mg}, 1.13 \mathrm{mmol}\right.$ ) was added and the reaction was kept under stirring at $90{ }^{\circ} \mathrm{C}$ for additional 25 hours. After this time the reaction reached $75 \%$ conversion. 


\section{E-Factor calculations}

Calculations has been aimed at the comparison of the two procedures based on the use of homogeneous or heterogeneous base

7a:

Homogeneous: (769 mg \{reaction solvent $\}+114 \mathrm{mg}\{\mathbf{2}\}+196 \mathrm{mg}\{\mathbf{5} \mathbf{a}\}+147 \mathrm{mg}\{\mathbf{6} \mathbf{a}\}+818 \mathrm{mg}$ \{azeotrope for washing $\}+4485 \mathrm{mg}\{$ EtOAc $\}+4500 \mathrm{mg}\left\{\mathrm{H}_{2} \mathrm{O}\right\}+1500 \mathrm{mg}\left\{\mathrm{Na}_{2} \mathrm{SO}_{4}\right\}-137 \mathrm{mg}$ \{product\}) $/ 137 \mathrm{mg}=\mathbf{9 0}$ (without considering chromatographic purification)

Heterogeneous: $(196 \mathrm{mg}\{\mathbf{5 a}\}+245 \mathrm{mg}\{\mathbf{6} \mathbf{a}\}+143 \mathrm{mg}\{\mathbf{2}$ used for regeneration of $\mathbf{8}\}+120 \mathrm{mg}$ $\{5 \%$ of azeotrope not recovered by distillation\} $+1636 \mathrm{mg}$ \{azeotrope for washing $-129 \mathrm{mg}$ \{product\})/129 mg = 17 (without considering chromatographic purification)

7b:

Homogeneous: (769 mg \{reaction solvent $\}+114 \mathrm{mg}\{\mathbf{2}\}+236 \mathrm{mg}\{\mathbf{5 b}\}+147 \mathrm{mg}\{\mathbf{6} \mathbf{a}\}+818 \mathrm{mg}$ $\{$ azeotrope for washing $\}+4485 \mathrm{mg}\{$ EtOAc $\}+4500 \mathrm{mg}\left\{\mathrm{H}_{2} \mathrm{O}\right\}+1500 \mathrm{mg}\left\{\mathrm{Na}_{2} \mathrm{SO}_{4}\right\}-208 \mathrm{mg}$ $\{$ product $\}) / 208 \mathrm{mg}=\mathbf{5 9}$

7c:

Homogeneous: $(769 \mathrm{mg}\{$ reaction solvent $\}+114 \mathrm{mg}\{\mathbf{2}\}+254 \mathrm{mg}\{\mathbf{5 c}\}+147 \mathrm{mg}\{\mathbf{6 a}\}+818 \mathrm{mg}$ $\{$ azeotrope for washing $\}+4485 \mathrm{mg}\{$ EtOAc $\}+4500 \mathrm{mg}\left\{\mathrm{H}_{2} \mathrm{O}\right\}+1500 \mathrm{mg}\left\{\mathrm{Na}_{2} \mathrm{SO}_{4}\right\}-218 \mathrm{mg}$ \{product\}) $/ 218 \mathrm{mg}=57$

Heterogeneous: (254 mg $\{\mathbf{5 c}\}+147 \mathrm{mg}\{\mathbf{6} \mathbf{a}\}+143 \mathrm{mg}\{\mathbf{2}$ used for regeneration of $\mathbf{8}\}+120 \mathrm{mg}$ $\{5 \%$ of azeotrope not recovered by distillation $+1636 \mathrm{mg}$ \{azeotrope for washing $-207 \mathrm{mg}$ \{product\} $) / 207 \mathrm{mg}=\mathbf{1 0}$

7d:

Homogeneous: (769 mg \{reaction solvent $\}+114 \mathrm{mg}\{\mathbf{2}\}+236 \mathrm{mg}\{\mathbf{5 d}\}+147 \mathrm{mg}\{\mathbf{6} \mathbf{a}\}+818 \mathrm{mg}$ azeotrope for washing $\}+4485 \mathrm{mg}\{\mathrm{EtOAc}\}+4500 \mathrm{mg}\left\{\mathrm{H}_{2} \mathrm{O}\right\}+1500 \mathrm{mg}\left\{\mathrm{Na}_{2} \mathrm{SO}_{4}\right\}-199 \mathrm{mg}$ \{product\})/199 $\mathrm{mg}=62$

Heterogeneous: $(236 \mathrm{mg}\{\mathbf{5 d}\}+147 \mathrm{mg}\{\mathbf{6 a}\}+143 \mathrm{mg}\{\mathbf{2}$ used for regeneration of $\mathbf{8}\}+120 \mathrm{mg}$ $\{5 \%$ of azeotrope not recovered by distillation $+1636 \mathrm{mg}$ \{azeotrope for washing $-197 \mathrm{mg}$ $\{$ product $\}) / 197 \mathrm{mg}=\mathbf{1 1}$

7e:

Homogeneous: (769 mg \{reaction solvent $\}+114 \mathrm{mg}\{\mathbf{2}\}+207 \mathrm{mg}\{\mathbf{5 e}\}+147 \mathrm{mg}\{\mathbf{6} \mathbf{a}\}+818 \mathrm{mg}$ \{azeotrope for washing $\}+4485 \mathrm{mg}\{$ EtOAc $\}+4500 \mathrm{mg}\left\{\mathrm{H}_{2} \mathrm{O}\right\}+1500 \mathrm{mg}\left\{\mathrm{Na}_{2} \mathrm{SO}_{4}\right\}-155 \mathrm{mg}$ \{product\}) $/ 155 \mathrm{mg}=\mathbf{8 0}$ (without considering chromatographic purification)

Heterogeneous: (207 mg $\{\mathbf{5 e}\}+245 \mathrm{mg}\{\mathbf{6} \mathbf{a}\}+143 \mathrm{mg}\{\mathbf{2}$ used for regeneration of $\mathbf{8}\}+120 \mathrm{mg}$ $\{5 \%$ of azeotrope not recovered by distillation $+1636 \mathrm{mg}$ \{azeotrope for washing $-112 \mathrm{mg}$ \{product\}) $/ 112 \mathrm{mg}=\mathbf{2 0}$ (without considering chromatographic purification)

7f:

Homogeneous: $(769 \mathrm{mg}$ \{reaction solvent $\}+114 \mathrm{mg}\{\mathbf{2}\}+222 \mathrm{mg}\{\mathbf{5 f}\}+147 \mathrm{mg}\{\mathbf{6 a}\}+818 \mathrm{mg}$ \{azeotrope for washing $\}+4485 \mathrm{mg}\{$ EtOAc $\}+4500 \mathrm{mg}\left\{\mathrm{H}_{2} \mathrm{O}\right\}+1500 \mathrm{mg}\left\{\mathrm{Na}_{2} \mathrm{SO}_{4}\right\}-147 \mathrm{mg}$ \{product\}) $/ 147 \mathrm{mg}=\mathbf{8 4}$ (without considering chromatographic purification)

7g:

Homogeneous: $(769 \mathrm{mg}$ \{reaction solvent $\}+114 \mathrm{mg}\{\mathbf{2}\}+209 \mathrm{mg}\{\mathbf{5 g}\}+147 \mathrm{mg}\{\mathbf{6} \mathbf{a}\}+818 \mathrm{mg}$ \{azeotrope for washing $\}+4485 \mathrm{mg}\{$ EtOAc $\}+4500 \mathrm{mg}\left\{\mathrm{H}_{2} \mathrm{O}\right\}+1500 \mathrm{mg}\left\{\mathrm{Na}_{2} \mathrm{SO}_{4}\right\}-117 \mathrm{mg}$ \{product\}) $/ 117 \mathrm{mg}=\mathbf{1 0 6}$ (without considering chromatographic purification)

7h: 
Homogeneous: $(769 \mathrm{mg}$ \{reaction solvent $\}+114 \mathrm{mg}\{\mathbf{2}\}+201 \mathrm{mg}\{\mathbf{5 h}\}+147 \mathrm{mg}\{\mathbf{6 a}\}+818 \mathrm{mg}$ azeotrope for washing $\}+4485 \mathrm{mg}\{\mathrm{EtOAc}\}+4500 \mathrm{mg}\left\{\mathrm{H}_{2} \mathrm{O}\right\}+1500 \mathrm{mg}\left\{\mathrm{Na}_{2} \mathrm{SO}_{4}\right\}-130 \mathrm{mg}$ \{product\}) $/ 130 \mathrm{mg}=\mathbf{9 5}$ (without considering chromatographic purification)

$7 \mathbf{i}:$

Homogeneous: $(769 \mathrm{mg}\{$ reaction solvent $\}+114 \mathrm{mg}\{\mathbf{2}\}+236 \mathrm{mg}\{\mathbf{5 i}\}+247 \mathrm{mg}\{\mathbf{6 b}\}+818 \mathrm{mg}$ \{azeotrope for washing $\}+4485 \mathrm{mg}\{$ EtOAc $\}+4500 \mathrm{mg}\left\{\mathrm{H}_{2} \mathrm{O}\right\}+1500 \mathrm{mg}\left\{\mathrm{Na}_{2} \mathrm{SO}_{4}\right\}-246 \mathrm{mg}$ \{product\})/246 $\mathrm{mg}=\mathbf{5 0}$

Heterogeneous: (239 $\mathrm{mg}\{\mathbf{5 i}\}+247 \mathrm{mg}\{\mathbf{6 b}\}+143 \mathrm{mg}\{\mathbf{2}$ used for regeneration of $\mathbf{8}\}+120 \mathrm{mg}$ $\{5 \%$ of azeotrope not recovered by distillation $\}+1636 \mathrm{mg}$ \{azeotrope for washing $-249 \mathrm{mg}$ \{product $\}) / 249 \mathrm{mg}=\mathbf{9}$

7j:

Homogeneous: (769 mg \{reaction solvent $\}+114 \mathrm{mg}\{\mathbf{2}\}+236 \mathrm{mg}\{\mathbf{5 d}\}+247 \mathrm{mg}\{\mathbf{6 b}\}+818 \mathrm{mg}$ azeotrope for washing $\}+4485 \mathrm{mg}\{\mathrm{EtOAc}\}+4500 \mathrm{mg}\left\{\mathrm{H}_{2} \mathrm{O}\right\}+1500 \mathrm{mg}\left\{\mathrm{Na}_{2} \mathrm{SO}_{4}\right\}-241 \mathrm{mg}$ \{product\}) $/ 241 \mathrm{mg}=\mathbf{5 2}$

Heterogeneous: $(236 \mathrm{mg}\{\mathbf{5 d}\}+247 \mathrm{mg}\{\mathbf{6 b}\}+143 \mathrm{mg}\{\mathbf{2}$ used for regeneration of $\mathbf{8}\}+120 \mathrm{mg}$ $\{5 \%$ of azeotrope not recovered by distillation $+1636 \mathrm{mg}$ \{azeotrope for washing $-255 \mathrm{mg}$ \{product $\}) / 255 \mathrm{mg}=\mathbf{8}$

7k:

Homogeneous: $(769 \mathrm{mg}$ \{reaction solvent $\}+114 \mathrm{mg}\{\mathbf{2}\}+236 \mathrm{mg}\{\mathbf{5 i}\}+247 \mathrm{mg}\{\mathbf{6 c}\}+818 \mathrm{mg}$ \{azeotrope for washing $\}+4485 \mathrm{mg}\{\mathrm{EtOAc}\}+4500 \mathrm{mg}\left\{\mathrm{H}_{2} \mathrm{O}\right\}+1500 \mathrm{mg}\left\{\mathrm{Na}_{2} \mathrm{SO}_{4}\right\}-249 \mathrm{mg}$ \{product $\}) / 249 \mathrm{mg}=\mathbf{5 0}$

7I:

Heterogeneous: $(236 \mathrm{mg}\{\mathbf{5 i}\}+147 \mathrm{mg}\{\mathbf{6 a}\}+143 \mathrm{mg}\{\mathbf{2}$ used for regeneration of $\mathbf{8}\}+120 \mathrm{mg}$ $\{5 \%$ of azeotrope not recovered by distillation $+1636 \mathrm{mg}$ \{azeotrope for washing $-191 \mathrm{mg}$ \{product\} $) / 191 \mathrm{mg}=\mathbf{1 1}$ 
Figure S1. Solid state ${ }^{13} \mathrm{C}$ CP MAS NMR spectrum of material 1.

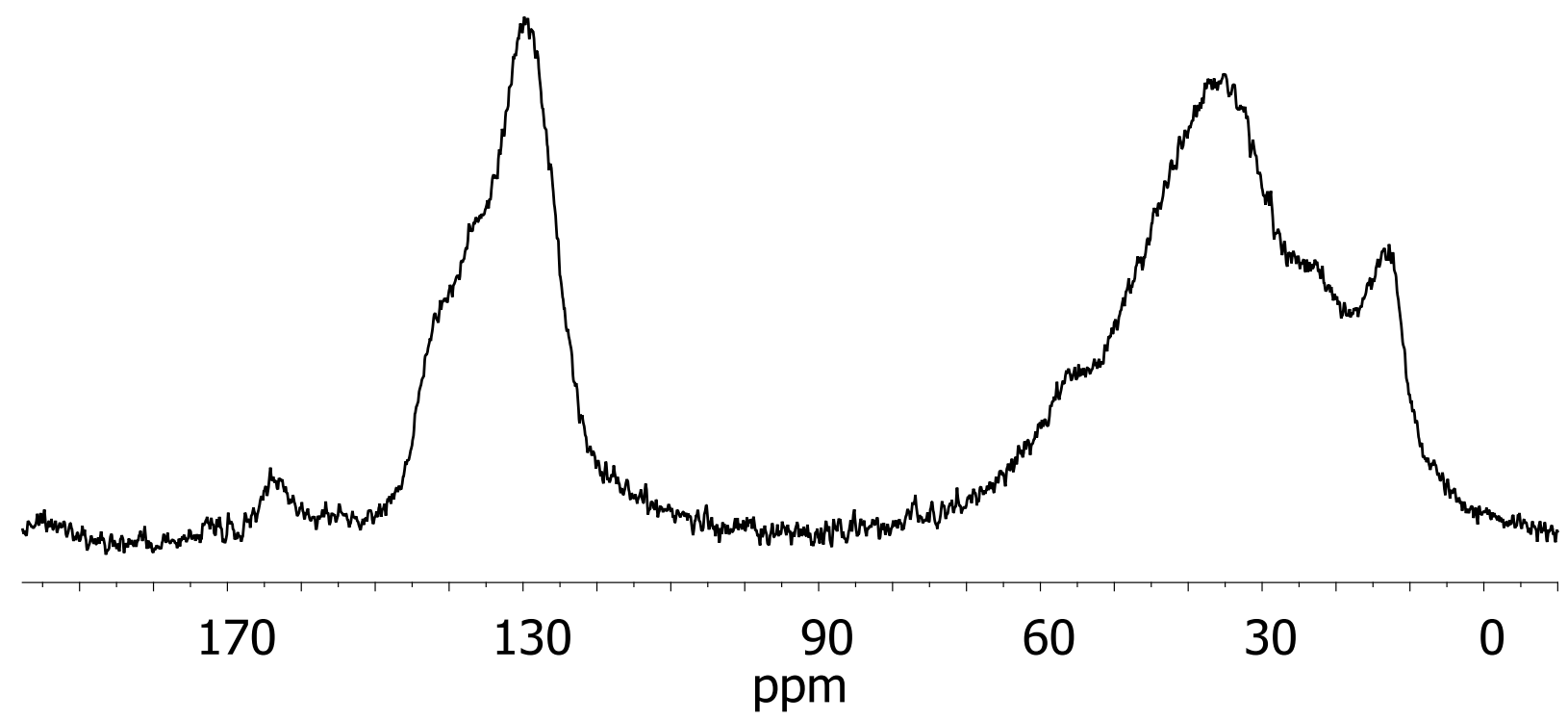

Figure S2. TEM images ( $a$ and $b)$ of material 1.

(a)

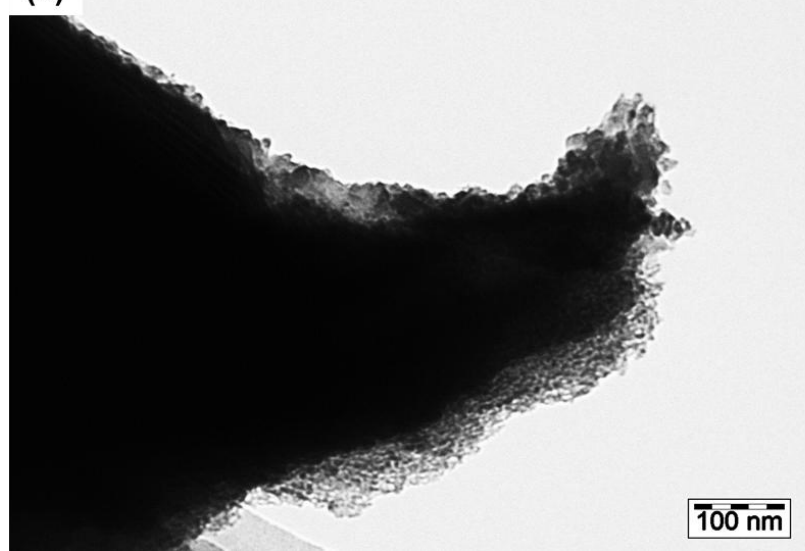

(b)

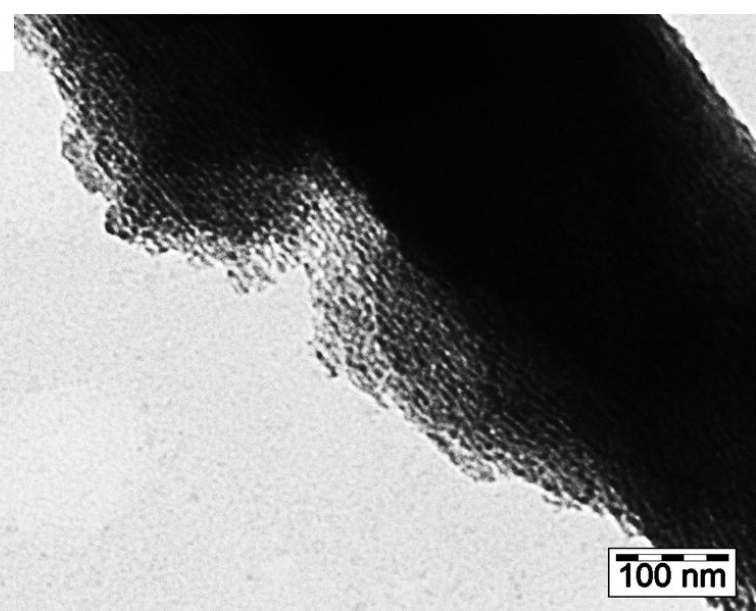

Pd nanoparticles are not clearly visible in the images due to the low content of $\operatorname{Pd}(0)$ in the material with the formation of small nanoparticles with a narrow particles size distribution. 
Figure S3. XPS spectrum Pd core $3 d$ of material 1. The core-level spectrum was referred to the $\mathrm{C} 1 \mathrm{~s}$ carbon peak at $284.6 \mathrm{eV}$.

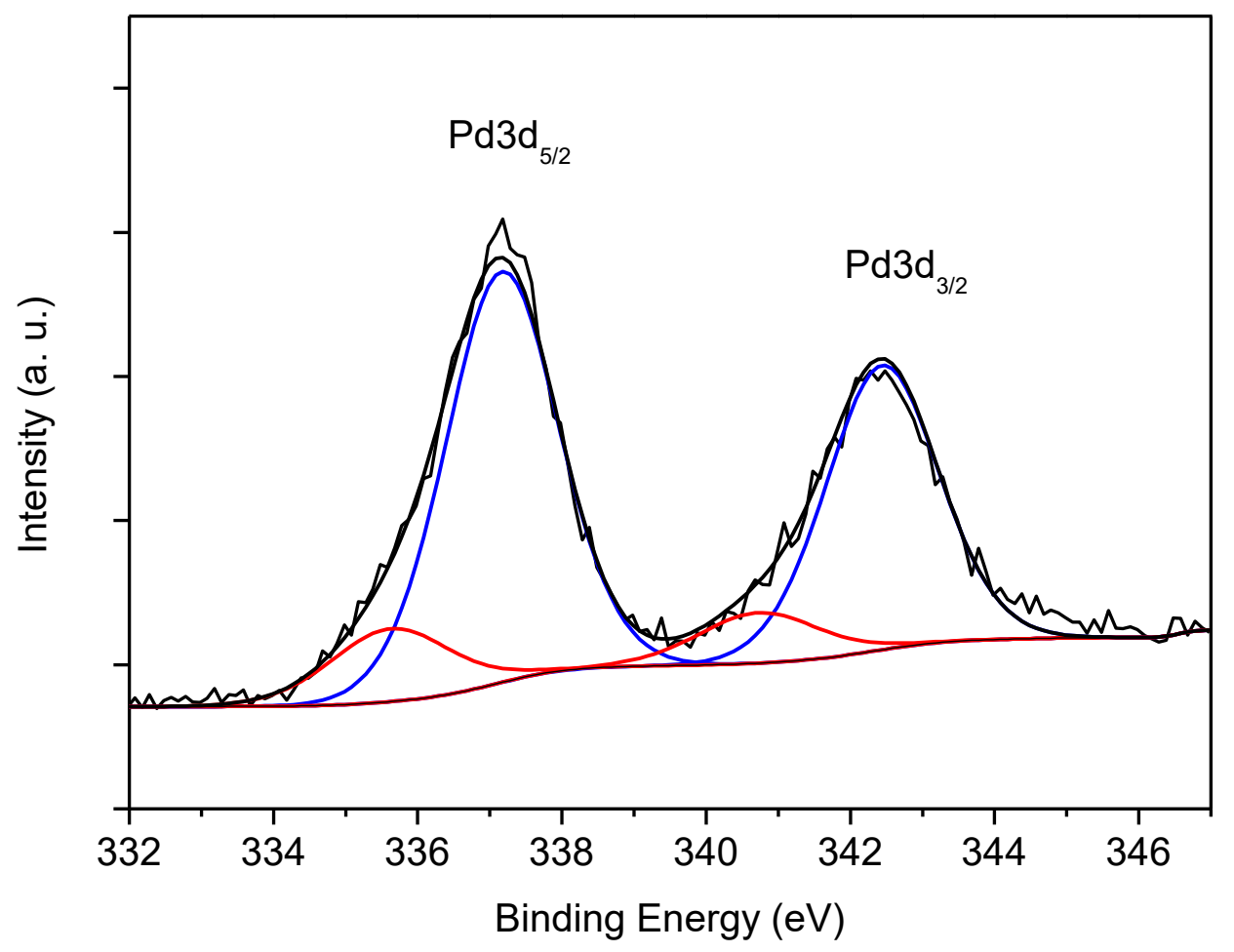

\begin{tabular}{|c|c|c|c|c|c|}
\hline Entry & Material & $\begin{array}{c}\mathrm{Pd} 3 \mathrm{~d}_{5 / 2} \mathrm{eV} \\
{\left[\mathrm{Pd}^{2+}\right]}\end{array}$ & $\begin{array}{c}\mathrm{Pd} 3 d_{5 / 2} \mathrm{eV} \\
{[\mathrm{Pd} 0]}\end{array}$ & $\begin{array}{c}\mathrm{Pd}(0) \\
{[\%]}\end{array}$ & $\mathrm{Pd} / \mathrm{C}$ \\
\hline 1 & 1 & 337.0 & 335.4 & 15 & 0.02 \\
\hline
\end{tabular}




\section{Characterization Data}

\begin{tabular}{l}
\hline Chem.Name \\
\hline List.Ref
\end{tabular}




\begin{tabular}{l}
\hline Chem.Name \\
\hline List.Ref
\end{tabular}




\begin{tabular}{|c|c|c|c|c|}
\hline Chem.Name & \multicolumn{4}{|c|}{ Methyl 4-(phenylethynyl)benzoate (7c) } \\
\hline List.Ref & \multicolumn{4}{|c|}{ Angew. Chem. Int. Ed. 2010, 49, $4067-4070$} \\
\hline \multicolumn{5}{|c|}{$\Longrightarrow \begin{array}{c}1(0.4 \mathrm{~mol} \%) \\
\text { Base }(1.2 \mathrm{eq})\end{array} \quad \mathrm{O}$} \\
\hline \multicolumn{5}{|l|}{$\begin{array}{l}\text { Homogeneous base: } \\
\text { Prepared according } \\
\mathrm{CH}_{3} \mathrm{CN} / \mathrm{H}_{2} \mathrm{O} \text { azeotro } \\
\text { methyl 4-iodobenzoa } \\
98 \% \text { purity). The pro }\end{array}$} \\
\hline \multicolumn{5}{|c|}{$\begin{array}{l}\text { Prepared according to the general procedure using: catalyst }(\mathbf{1})(10 \% \mathrm{wt} \text { of } \mathrm{Pd})(4 \mathrm{mg}, 0.4 \mathrm{mmol} \%) \text {, } \\
\mathrm{CH}_{3} \mathrm{CN} / \mathrm{H}_{2} \mathrm{O} \text { azeotrope }(0.94 \mathrm{~mL}, 84 / 16 \mathrm{wt} \%) \text {, PS-piperazine }(\mathbf{8})(335 \mathrm{mg}, 1.13 \mathrm{mmol}, 3.37 \mathrm{mmol} / \mathrm{g}) \text {, } \\
\text { methyl 4-iodobenzoate }(\mathbf{5 c})(254 \mathrm{mg}, 0.94 \mathrm{mmol}, 97 \% \text { purity), phenylacetylene }(\mathbf{6 a})(147 \mathrm{mg}, 1.41 \mathrm{mmol} \text {, } \\
98 \% \text { purity). The product (7c) was obtained as a pale yellow solid (207 } \mathrm{mg}, 93 \% \text { yield). }\end{array}$} \\
\hline Mol Formula & $\mathrm{C}_{16} \mathrm{H}_{12} \mathrm{O}_{2}$ & m.p. & $120-121^{\circ} \mathrm{C}$ & \\
\hline \multicolumn{5}{|c|}{ Elemental Analysis: C:81.34, H:5.12; found: C:81.90, H:5.22 } \\
\hline \multirow{6}{*}{$\begin{array}{l}{ }^{1} \mathrm{H} \mathrm{NMR} 400 \mathrm{MHz} \\
\mathrm{CDCl}_{3}\end{array}$} & $\delta$ value: & No. $\mathrm{H}$ & Mult & $\mathrm{J}$ value/Hz \\
\hline & 3.93 & 3 & $\mathrm{~s}$ & \\
\hline & 7.36 & 3 & br & \\
\hline & 7.55 & 2 & $\mathrm{~d}$ & 3.5 \\
\hline & 7.59 & 2 & $\mathrm{~d}$ & 8.2 \\
\hline & 8.02 & 2 & \begin{tabular}{l|l|} 
& $\mathrm{d}$ \\
\end{tabular} & 8.2 \\
\hline \multicolumn{5}{|c|}{$\begin{array}{l}{ }^{13} \mathrm{C} \mathrm{NMR}\left(100.6 \mathrm{~Hz}, \mathrm{CDCl}_{3}\right) \delta: 52.33,88.77,92.50,122.86,128.15,128.56,128.88,129.65,131.63 \\
131.87,166.67\end{array}$} \\
\hline \multicolumn{5}{|c|}{$\begin{array}{l}\text { GC-EIMS (m/z, \%): } 237\left(\mathrm{M}^{+}+1,17\right), 236\left(\mathrm{M}^{+}, 93\right), 206 \text { (16), } 205 \text { (100), } 177 \text { (25), } 176 \text { (48), } 151 \text { (18), } 150 \\
(13), 88 \text { (12). }\end{array}$} \\
\hline
\end{tabular}




\begin{tabular}{l}
\hline Chem.Name \\
\hline List.Ref
\end{tabular}




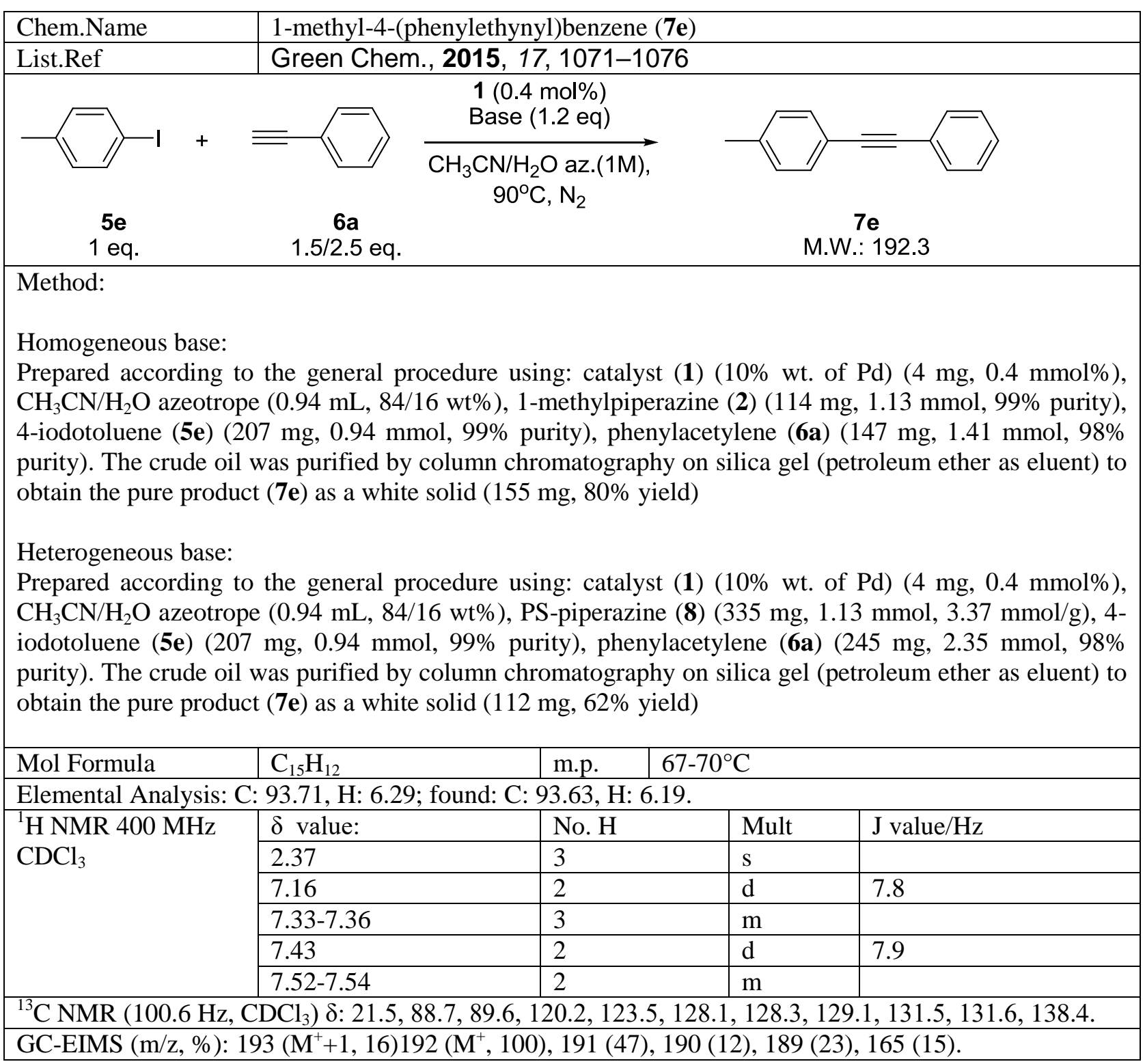




\begin{tabular}{l}
\hline Chem.Name \\
\hline List.Ref
\end{tabular}




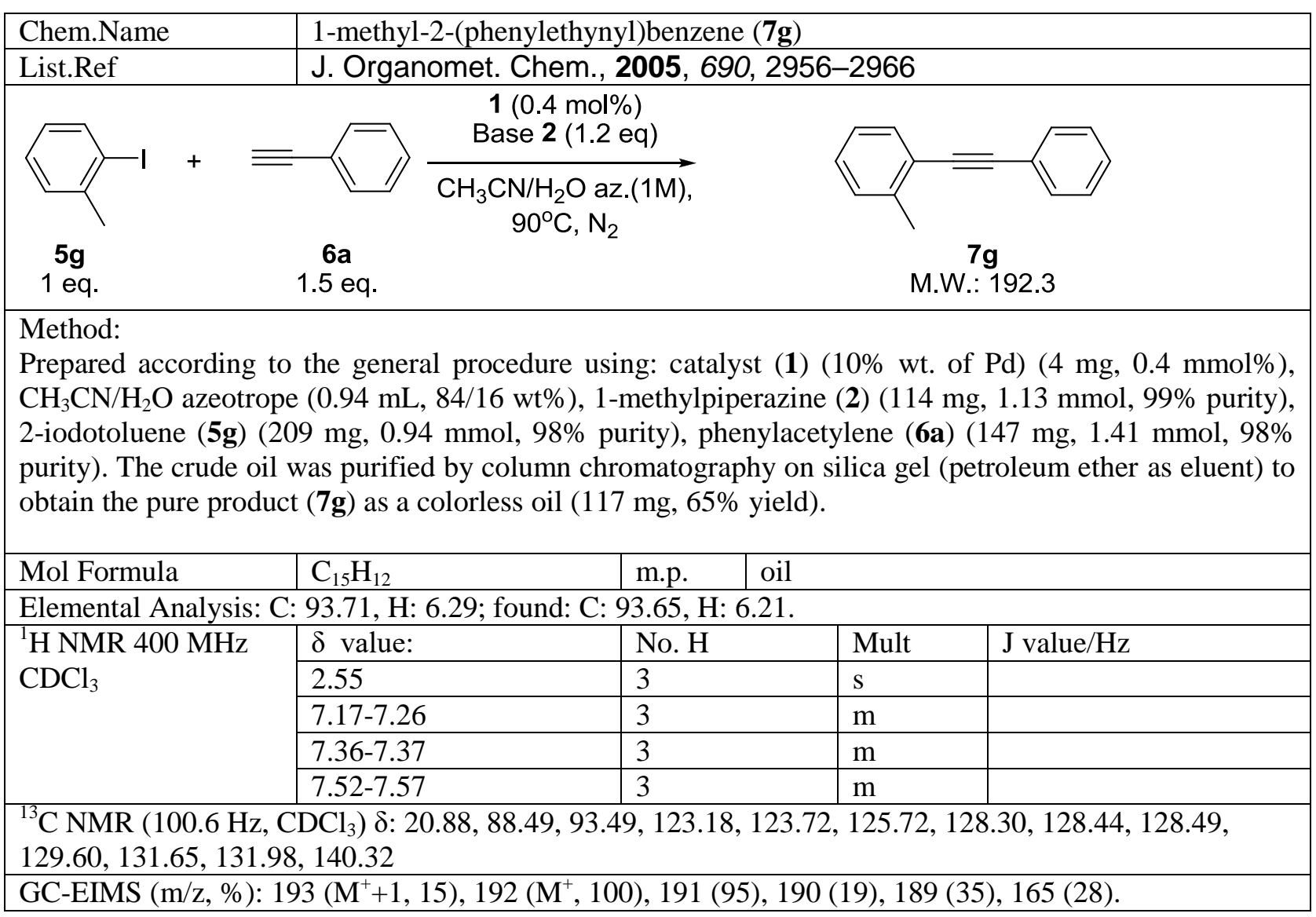




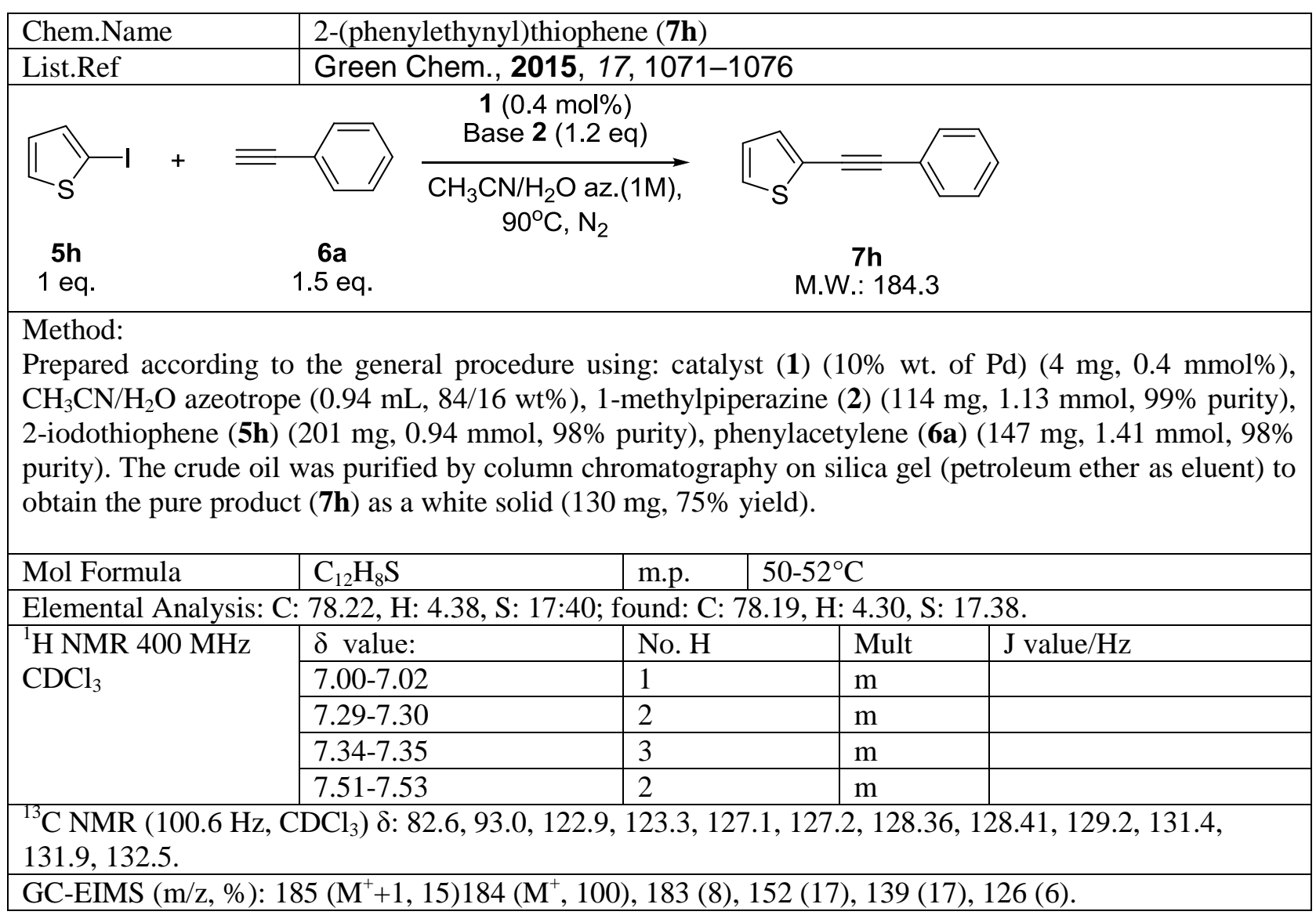




\begin{tabular}{|c|c|c|c|c|}
\hline Chem.Name & \multirow{2}{*}{\multicolumn{4}{|c|}{$\begin{array}{l}\text { 1-((4-nitrophenyl)ethynyl)-2-(trifluoromethyl)benzene (7i) } \\
\text { Chem. Eur. J.. 2008. 14.6994-6999 }\end{array}$}} \\
\hline List.Ref & & & & \\
\hline \multicolumn{5}{|c|}{ 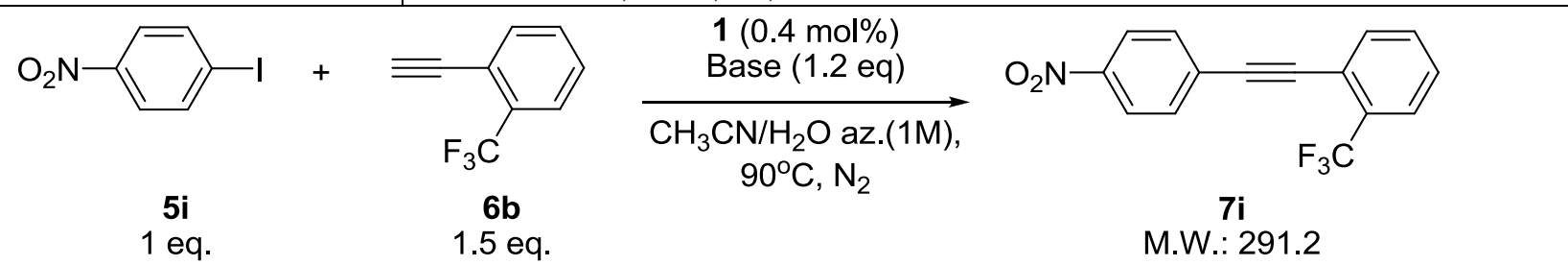 } \\
\hline \multicolumn{5}{|l|}{ Method: } \\
\hline \multicolumn{5}{|l|}{$\begin{array}{l}\text { Homogeneous base: } \\
\text { Prepared according } \mathrm{t} \\
\mathrm{CH}_{3} \mathrm{CN} / \mathrm{H}_{2} \mathrm{O} \text { azeotrol } \\
\text { 1-iodo-4-nitrobenzen } \\
\mathrm{mg}, 1.41 \mathrm{mmol}, 97 \%\end{array}$} \\
\hline \multicolumn{5}{|c|}{$\begin{array}{l}\text { Heterogeneous base: } \\
\text { Prepared according to the general procedure using: catalyst }(\mathbf{1})(10 \% \mathrm{wt} \text {. of Pd) }(4 \mathrm{mg}, 0.4 \mathrm{mmol} \%) \text {, } \\
\mathrm{CH}_{3} \mathrm{CN} / \mathrm{H}_{2} \mathrm{O} \text { azeotrope }(0.94 \mathrm{~mL}, 84 / 16 \mathrm{wt} \%) \text {, PS-piperazine }(\mathbf{8})(335 \mathrm{mg}, 1.13 \mathrm{mmol}, 3.37 \mathrm{mmol} / \mathrm{g}), 1- \\
\text { iodo-4-nitrobenzene }(\mathbf{5 i})(239 \mathrm{mg}, 0.94 \mathrm{mmol}, 98 \% \text { purity), } 2 \text {-ethynyl- } \alpha, \alpha, \alpha \text {-trifluorotoluene }(\mathbf{6 b})(247 \\
\mathrm{mg}, 1.41 \mathrm{mmol}, 97 \% \text { purity). The product (7i) was obtained as a pale yellow solid ( } 249 \mathrm{mg}, 91 \% \text { yield). }\end{array}$} \\
\hline Mol Formula & $\mathrm{C}_{15} \mathrm{H}_{8} \mathrm{~F}_{3} \mathrm{NO}_{2}$ & m.p. & $95^{\circ} \mathrm{C}$ & \\
\hline \multicolumn{5}{|c|}{ Elemental Analysis: C: 61.86, H: 2.77, N: 4.81; found: C: 61.76, H: $2.70, \mathrm{~N}: 4.79}$. \\
\hline \multirow{5}{*}{$\begin{array}{l}{ }_{1}^{1} \mathrm{H} \mathrm{NMR} 400 \mathrm{MHz} \\
\mathrm{CDCl}_{3}\end{array}$} & $\delta$ value: & No. $\mathrm{H}$ & Mult & $\mathrm{J}$ value $/ \mathrm{Hz}$ \\
\hline & $7.47-7.51$ & 1 & $\mathrm{~m}$ & \\
\hline & 7.54-7.58 & 1 & $\mathrm{~m}$ & \\
\hline & $7.66-7.73$ & 4 & $\mathrm{~m}$ & \\
\hline & 8.23 & 2 & $\mathrm{~d}$ & 8.5 \\
\hline \multirow{2}{*}{\multicolumn{5}{|c|}{$\begin{array}{l}{ }^{13} \mathrm{C} \mathrm{NMR}\left(100.6 \mathrm{~Hz}, \mathrm{CDCl}_{3}\right) \delta: 90.6,93.0,120.7,123.5\left(\mathrm{q}, \mathrm{J}_{\mathrm{F}-\mathrm{C}}=273.5 \mathrm{~Hz}\right), 123.8,126.3,129.4,129.9, \\
131.9,132.3,132.8,134.4,147.7 . \\
{ }^{19} \mathrm{~F} \mathrm{NMR}\left(376.4 \mathrm{~Hz} \mathrm{CDCl}_{2}\right) \delta \cdot-62.6\end{array}$}} \\
\hline & & & & \\
\hline \multicolumn{5}{|c|}{$\begin{array}{l}\text { GC-EIMS (m/z, \%): } 292\left(\mathrm{M}^{+}+1,16\right), 291\left(\mathrm{M}^{+}, 100\right), 261(22), 245(19), 243(11), 233(30), 232(30), 225 \\
(39), 214(13) .\end{array}$} \\
\hline
\end{tabular}




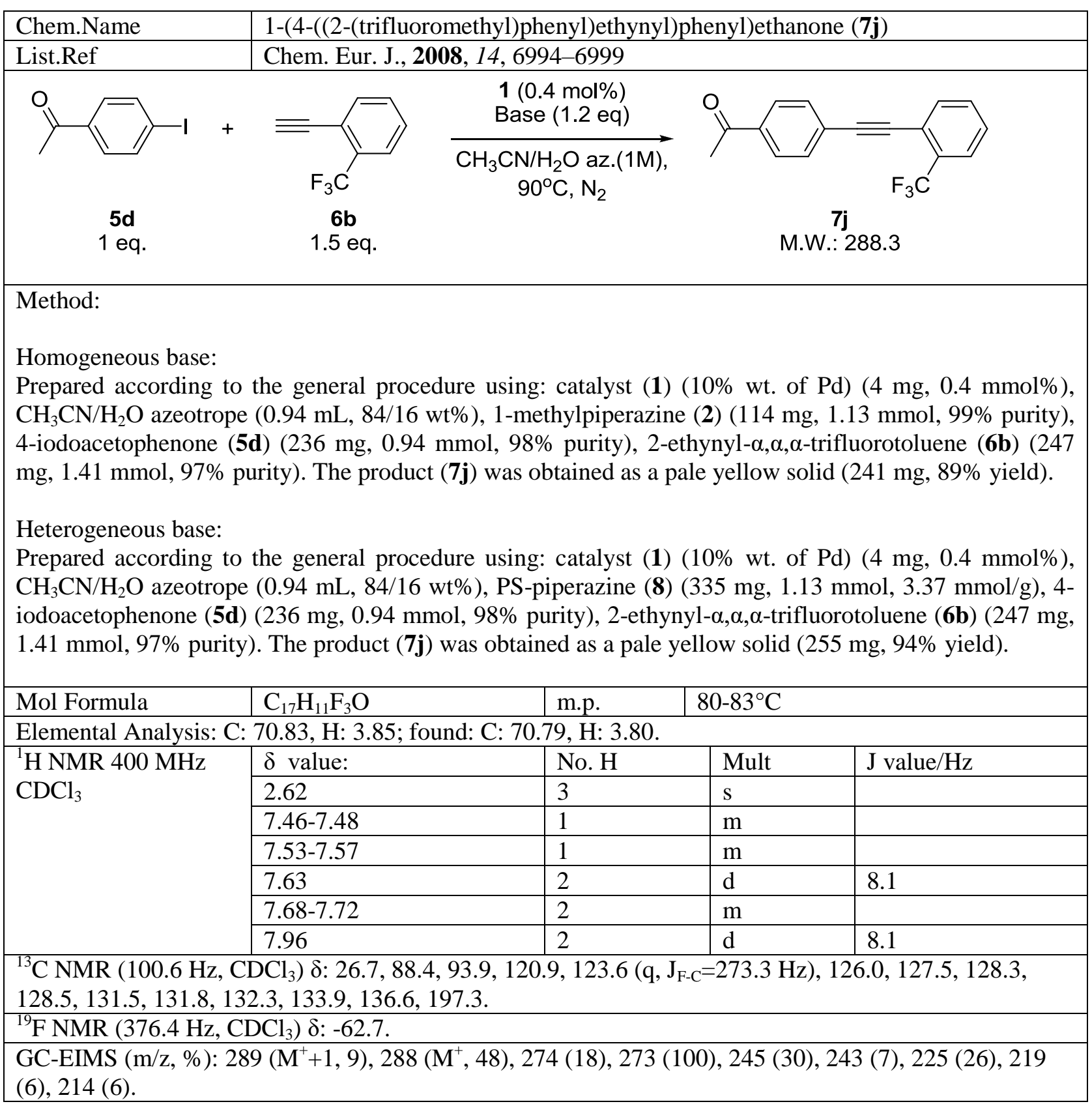




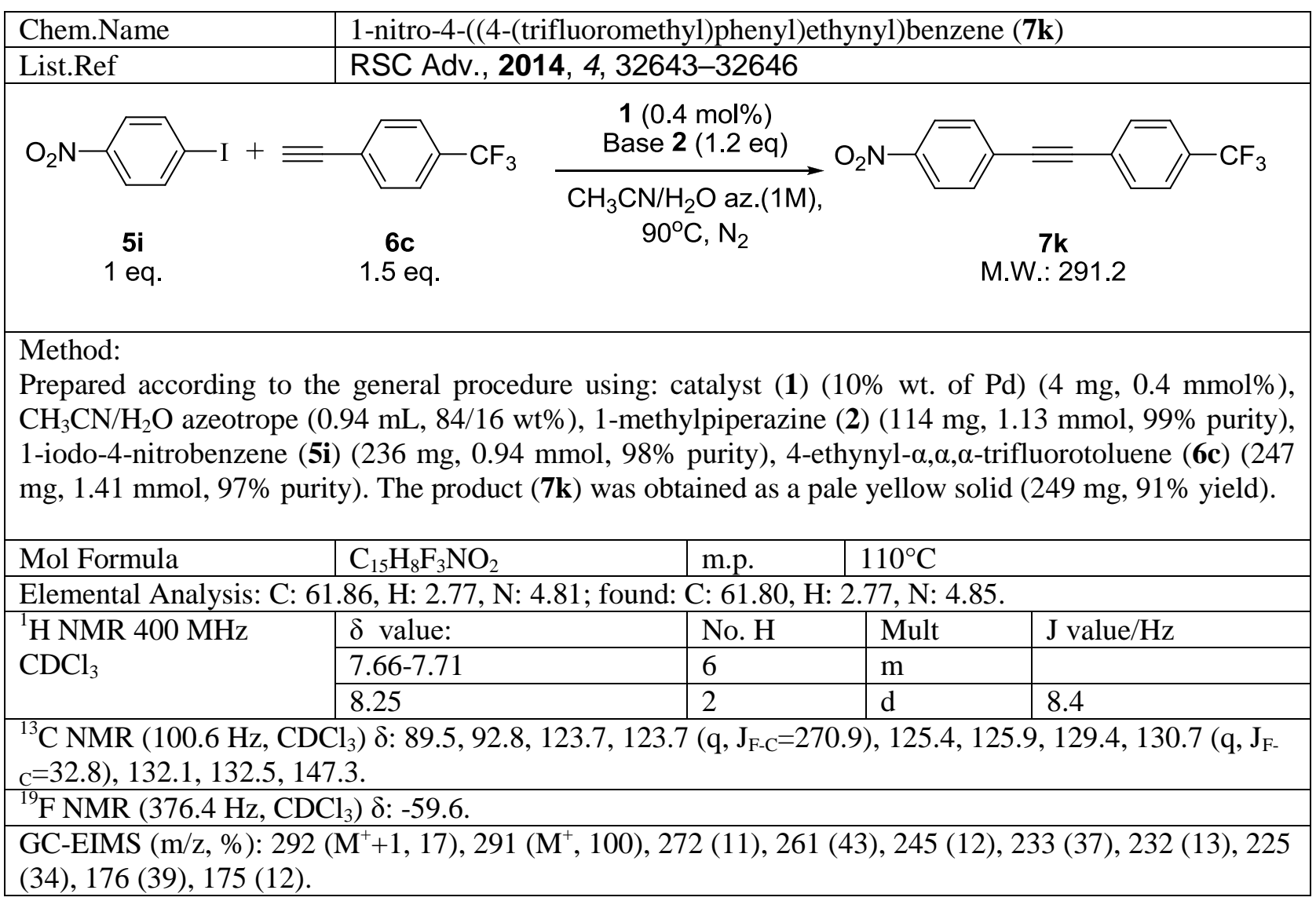




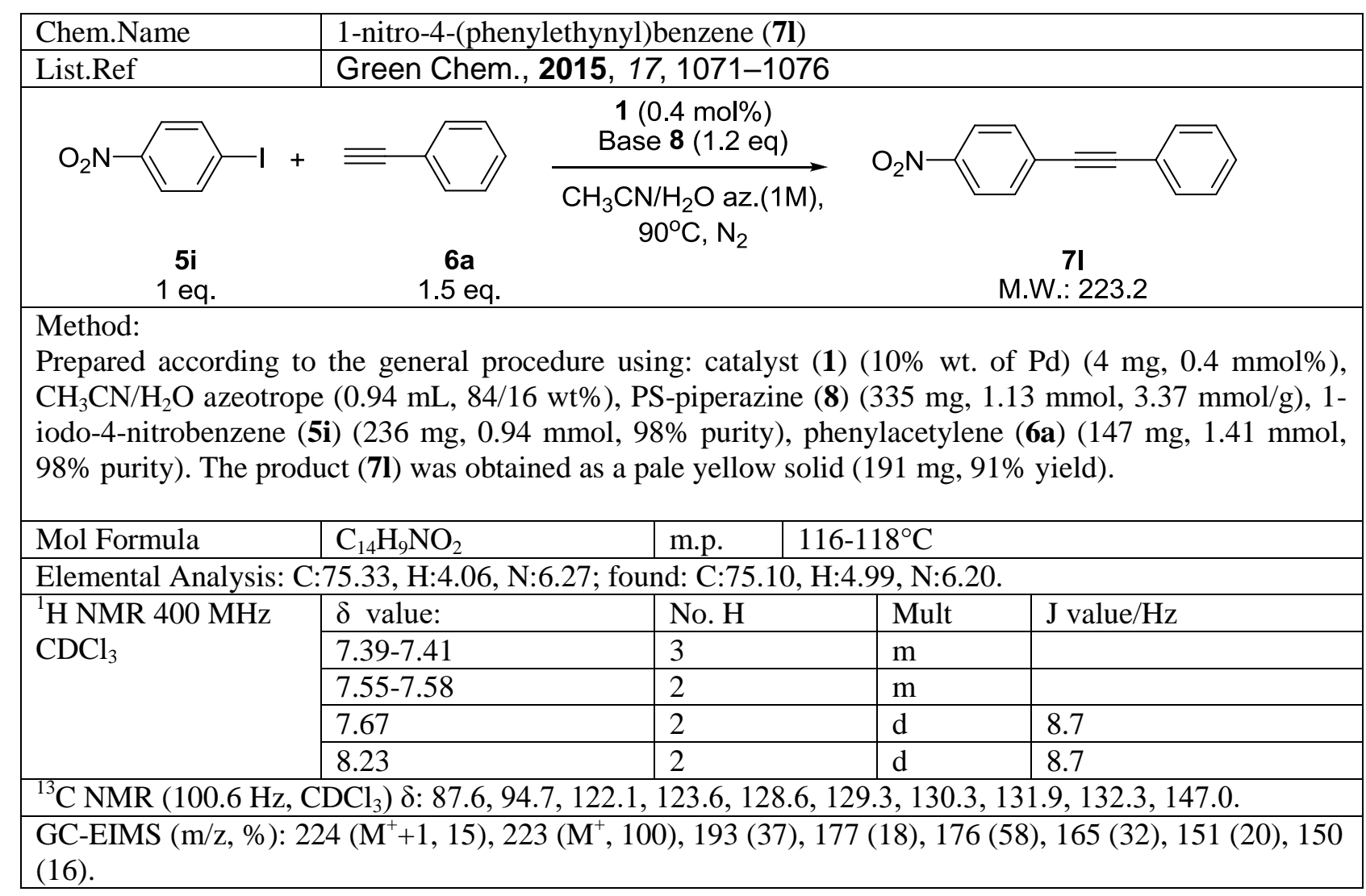




Chem.Name
Method:
Merrifield resin $(4 \mathrm{~g}, 200-400$ mesh, $3.5-4.5$ mmol/g $\mathrm{Cl} \mathrm{loading}, 1 \%$ cross-linked with
divinylbenzene) was reacted with an excess of piperazine $(10 \mathrm{~g}, 0.115$ mol, $99 \%$ purity) in toluene
(40 $\mathrm{mL})$ at $60^{\circ} \mathrm{C}$ for 72 hours. The resin was washed with a $0.5 \mathrm{M}$ of piperazine in toluene, then
toluene, water and acetone to remove the excess of amine and finally dried under vacuum. The amine
loading $(3.37 \mathrm{mmol} / \mathrm{g})$ was determined by elemental analysis.

